\title{
Electrical and photoelectrical properties of iodine modified porous silicon on silicon substrates
}

\author{
I.B. Olenych \\ Ivan Franko Lviv National University, Department of Electronics, \\ 50, Dragomanov str., 79005 Lviv, Ukraine \\ Phone: 38 (032) 239-46-23; e-mail: iolenych@gmail.com
}

\begin{abstract}
Current-voltage characteristics, spectral dependences of photovoltage and short-circuit current of the structures based on porous silicon at adsorption of iodine molecules are presented. It is revealed widening the spectral range of photosensitivity in the samples in short-wavelength range as compared with that of single crystal silicon. Kinetics of the photovoltage response inherent to the porous silicon-silicon structures has been investigated. The results are explained in the frame of qualitative model that involves formation of $p$ - $n$-transitions in these structures as a result of inversion of the conductivity type in porous silicon nanocrystals under the influence of adsorption of molecular iodine.
\end{abstract}

Keywords: porous silicon, iodine adsorption, photovoltage, photocurrent, spectral characteristics.

Manuscript received 15.05.12; revised version received 08.08.12; accepted for publication 17.10.12; published online 12.12.12.

\section{Introduction}

Porous silicon (PS) attracts attention of researchers because of its wide range of possible applications $[1,2]$. Due to its antireflective properties, a large area of absorbing surface, the increased width of the band gap of silicon nanocrystals there are interesting perspectives of application of PS in photoelectronics [3-7]. It has been also found that adsorption of chemically active or polar molecules causes a significant change in the concentration of free charge carriers in the PS and its electrophysical parameters [8-12]. It is assumed [8] that adsorption by silicon surface of $I_{2}$ molecules, which in this case reveal the acceptor properties, may lead to inversion of the electronic type of conductivity in silicon nanocrystals to the hole one. However, by now it does not pay sufficient attention to application of adsorption methods for controlling the conductivity type. So, the purpose of this study was to develop the method of "adsorption doping" of PS $-n$-Si barrier structures and to study their photovoltaic properties.

\section{Experimental}

The experimental samples of PS were manufactured by photoelectrochemical etching $[13,14]$ in the galvanostatic mode on the single-crystal silicon substrate of the 400- $\mu \mathrm{m}$ thickness and crystallographic orientation (100). The substrates have $n$-type of conductivity with a specific resistance of $4.5 \mathrm{Ohm} \cdot \mathrm{cm}$. To obtain a homogeneous layer, using the thermovacuum method we previously deposited a film of silver with a thickness of about $1 \mu \mathrm{m}$ on the back surface of the substrate. This film also served as a contact for further measurements. As an electrolyte the ethanol solution of hydrofluoric acid with the volume ratio of components $\mathrm{HF}: \mathrm{C}_{2} \mathrm{H}_{5} \mathrm{OH}=1: 1$ was used. The anodic current density was $30 \mathrm{~mA} / \mathrm{cm}^{2}$ and etching time $-3 \mathrm{~min}$. Under these technological conditions, mesoporous silicon layers are formed. According to SEM and AFM studies of the porous layer, the thickness and size of silicon nanowires were about $8 \mu \mathrm{m}$ and $40 \mathrm{~nm}$, respectively. After electrochemical treatment, PS surface was rinsed with 
distilled water and dried in air stream at room temperature for $30 \mathrm{~min}$. These structures were divided on samples with an area of about $1 \mathrm{~cm}^{2}$. Adsorption of $I_{2}$ molecules was performed from 1,5 and $10 \%$ ethanol solution of iodine for different samples of PS, after that the samples were dried for $30 \mathrm{~min}$ at room temperature. Optical transparent clamping electrode (glass plate with conductive layer of $\mathrm{SnO}_{2}$ ) was served as a contact to the porous layer.

The measurement of current-voltage characteristics (CVC) of the structures based on PS was performed during the passage of current through the structure in the direction perpendicular to the surface. Photoelectrical phenomena were studied under illumination of the structures from a porous layer side perpendicularly to its surface by using a He-Ne laser $(\lambda=0.63 \mu \mathrm{m})$ with a radiation power of $2 \mathrm{~mW}$. Measurements of the spectral dependence of the photoresponse (in the regimes of photovoltage and photocurrent) were carried out using a standard optical equipment with a diffraction monochromator and filament lamp $(2800 \mathrm{~K})$. The spectra of photovoltage were normalized on the curve of blackbody radiation with a temperature of $2800 \mathrm{~K}$ and were corrected taking into account the spectral sensitivity of the setup. It was also measured spectral dependence of the photoresponse of an industrial silicon photodiode. To study the kinetics of photoresponse, light was modulated with a frequency of $350 \mathrm{~Hz}$ through electromechanical interruption of the beam and then was directed on the PS surface. All measurements were carried out at room temperature.

\section{Results and discussion}

The reference sample of PS $-n$-Si (not modified $\mathrm{I}_{2}$ ) had symmetrical and nonlinear current-voltage characteristics, indicating the existence of several potential barriers in this structure (Fig. 1). Adsorption of iodine molecules led to significant changes in electrical properties of structures of PS $-n$-Si. The observed rectifying nature of the $\mathrm{CVC}$ (the direct branch of CVC corresponds to the positive potential on the porous layer). It indicates dominance of one of the potential barriers. There observed increase in the concentration of iodine molecules in solution from which adsorption took place, it means that the number of adsorbed molecules resulted in increased rectification coefficient of CVC of the adsorptionally modified structures PS $-n$-Si.

Under the influence of light illumination of PS surface by He-Ne laser with an intensity of $60 \mathrm{~mW} / \mathrm{cm}^{2}$, the CVC of PS $-n$-Si sample with adsorbed iodine molecules changed similarly to those observed in a photodiode structure (Fig. 2). Moreover, increasing the number of adsorbed molecules resulted in an increased magnitude of photovoltage and photocurrent in the short-circuit regime.

Qualitatively, this change in CVC under the influence of adsorption of $\mathrm{I}_{2}$ can be explained as follows. Adsorption of molecules with acceptor properties on silicon surface leads to formation of the surface acceptor levels and appropriate bending of energy bands what, in its turn, can cause the inversion of conductivity type in the near-surface region of weakly doped $n$-Si [15]. In PS nanocrystals characterized by large specific surface inversion of the electronic type of conductivity to the hole one under the influence of adsorption of iodine molecules can be expected in the whole bulk of nanocrystals [8], similar to the observed inversion of the hole type conductivity of PS to the electronic one under the influence of adsorption of ammonia molecules [16]. As a result, formation of electrical barriers at the PS $-n$ Si boundary (similarly to $p$ - $n$-junctions in silicon diodes) was observed. So, the structure of adsorptionally modified PS $-n$-Si can be considered as parallelly arranged $p-n$-junctions (see Fig. 1, inset). However, we can not exclude the existence of electrical barriers of other nature (for example, non-linearity of contact) photoconduction of porous layer and, consequently, more complex mechanisms of current passage in these structures. Under the influence of illumination in the open circuit regime the photogenerated electron-hole pairs are separated by a potential barrier. During this process, the holes are accumulated in PS nanocrystals creating a positive potential on the porous layer with respect to the silicon substrate.

To obtain additional information about the nature of influence of the $\mathrm{I}_{2}$ adsorption on photoelectrical properties of porous silicon on silicon substrates, we have investigated spectral dependences of the photoresponse in these structures. The photovoltage spectra of adsorptionally modified samples at open circuit regime were similar to the photoresponse spectrum of industrial silicon photodiode and were characterized by the wide maximum in the 750 to $950 \mathrm{~nm}$ range (Fig. 3). In addition, it was observed expansion of the spectral photosensitivity of structures based on PS in the region of larger energies.

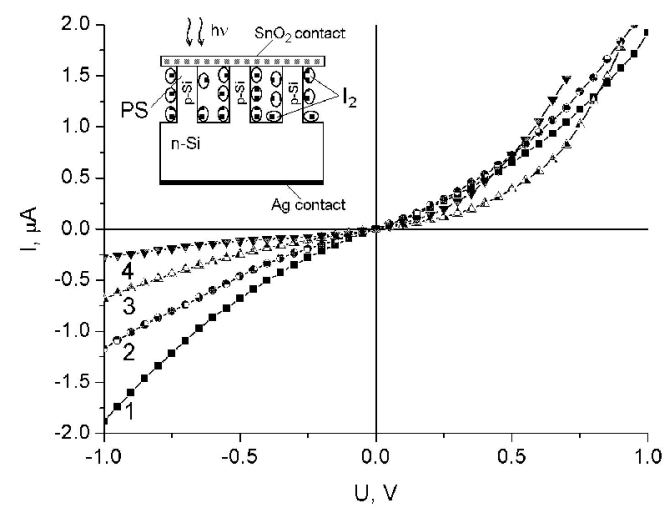

Fig. 1. Dark CVC of PS $-n$-Si structures: $1-$ without adsorption of $\mathrm{I}_{2} ; 2-\mathrm{I}_{2}$ adsorption was occurred from $1 \%$ solution in ethanol; $3-5 \% ; 4-10 \%$. Inset: schematic of the experimental samples when adsorbing $\mathrm{I}_{2}$.

\section{(C) 2012, V. Lashkaryov Institute of Semiconductor Physics, National Academy of Sciences of Ukraine}




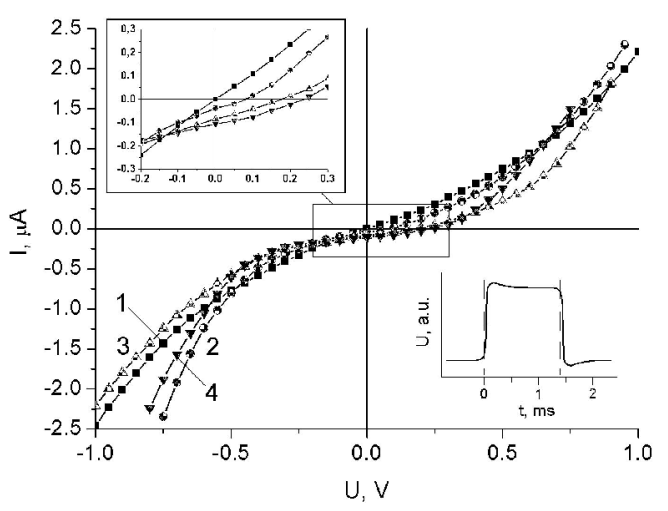

Fig. 2. CVC of PS $-n$-Si structures under illumination with He-Ne laser $(\lambda=0.63 \mu \mathrm{m})$ having the intensity $60 \mathrm{~mW} / \mathrm{cm}^{2}$ : 1 - without adsorption of $\mathrm{I}_{2} ; 2-\mathrm{I}_{2}$ adsorption was occurred from $1 \%$ solution in ethanol; $3-5 \% ; 4-10 \%$. Inset: Kinetics of photoresponse of PS $-n$-Si structure adsorptionally modified with iodine molecules on U-shaped pulse of light.

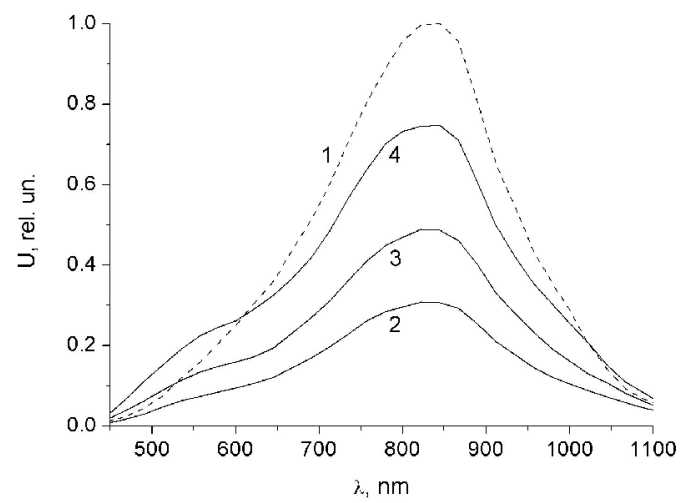

Fig. 3. Spectral dependence of photovoltage for industrial silicon photodiode (1) and structures of PS $-n$-Si $(2,3,4): 2-$ $\mathrm{I}_{2}$ adsorption was occurred from $1 \%$ solution in ethanol, $3-$ $5 \% ; 4-10 \%$.

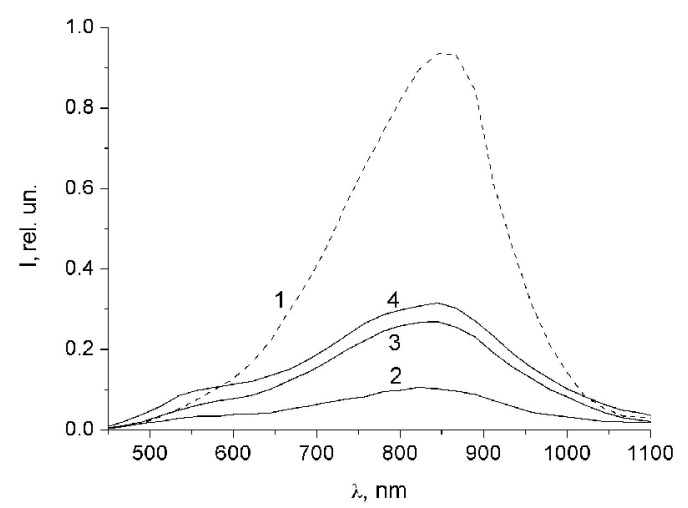

Fig. 4. Spectral dependence of photocurrent in the short circuit regime for industrial silicon photodiode $(1)$ and structures of PS $-n$-Si $(2,3,4): 2-\mathrm{I}_{2}$ was adsorption occurred from $1 \%$ solution in ethanol; $3-5 \% ; 4-10 \%$.
In the case of short-circuit regime, the character of spectral dependence of photocurrent corresponded to the photovoltage spectra (Fig. 4). The similarity of the spectral dependence of photoresponse of the PS $-n-\mathrm{Si}$ structure with that of silicon photodiode allows us to suggest that the photovoltage relates to the absorption of light in the silicon substrate and to the separation of photocarriers at the boundary of silicon - PS. Along with the positive sign of the photovoltage on porous layer, it can serve as an additional argument in favour of the proposed qualitative model of $p$ - $n$-junction formation due to inversion of the conductivity type of PS nanocrystals under the influence of iodine molecules.

The presence of a significant photoresponse of adsorptionally modified structures PS $-n$-Si allowed to explore the kinetics of the photovoltaic effect (see Fig. 2, inset). It was observed close values of the photovoltage increase and decay. Analysis of the photoresponse on light pulses of duration close to $1.4 \mathrm{~ms}$ gave an indication about complex generation-recombination and relaxation processes in these PS layers: there was a slight increase in photovoltage at the initial moment of a light pulse followed by relaxation for approximately $0.5 \mathrm{~ms}$.

It should be mentioned that desorption of iodine by PS is accompanied by decreasing the photovoltaic effect. So, endurance of the samples in normal atmospheric conditions for more than two days resulted in the disappearance of significant photosensitivity of the structures based on PS. Thus, the main condition for the photovoltage generation is adsorption of iodine molecules on PS nanostructures.

\section{Conclusions}

It has been shown that adsorption of $\mathrm{I}_{2}$ acceptor molecules modifies the electrical properties of freshly prepared PS nanostructures giving rise to formation of electrical barriers in the PS $-n$-Si structure. The obtained data suggest the possibility to control the conductivity type in PS nanocrystals by adsorption of chemically active molecules. The structures based on adsorptionally modified PS demonstrated the photovoltaic effect in the visible spectral range, which extends the prospects of their use as photodetectors. The obtained experimental results can be also used for the development of electronic devices not only based on porous silicon, but also on systems of semiconductor nanocrystals.

\section{Acknowledgement}

The author thanks to Prof. L.S. Monastyrskii, Dr. B.S. Sokolovskii and Prof. O.I. Aksimentyeva for discussion of the results.

\section{References}

1. H. Föll, M. Christophersen, J. Carstensen, G. Hasse, Formation and application of porous 
silicon // Mater. Sci. and Eng. Repts., 39(4), p. 93141 (2002).

2. O. Bisi, S. Ossicini, L. Pavesi, Porous silicon: a quantum sponge structure for silicon based optoelectronics // Surface Sci. Repts., 38(1), p. 1$126(2000)$.

3. K.Q. Peng, S.T. Lee, Silicon nanowires for photovoltaic solar energy conversion // Advanced Materials, 23(2), p. 198-215 (2011).

4. B. Ünal, A.N. Parbukov, S.C. Bayliss, Photovoltaic properties of a novel stain etched porous silicon and its application in photosensitive devices // Optical Materials, 17(1), p. 79-82 (2001).

5. L.V. Belyakov, D.N. Goryachev, O.M. Sreseli, Photoresponse and electroluminescence of silicon〈porous silicon〉-〈chemically deposited metal〉 structures // Semiconductors, 34(11), p. 1334-1337 (2000).

6. V.E. Primachenko, Ja.F. Kononets, B.M. Bulakh, E.F. Venger, E.B. Kaganovich, I.M. Kizyak, S.I. Kirillova, E.G. Manoilov, Yu.A. Tsyrkunov, The electronic and emissive properties of Au-doped porous silicon // Semiconductors, 39(5), p. 565-571 (2005).

7. A.V. Brodovoi, V.A. Brodovoi, V.A. Skryshevskyi, S.G. Bunchuk, L.M. Khnorozok, Photoelectric properties of metal-porous silicon-silicon planar heterostructures // Semiconductor Physics, Quantum Electronics and Optoelectronics, 5(4), p. 395-397 (2002).

8. A.S. Vorontsov, L.A. Osminkina, A.E. Tkachenko, E.A. Konstantinova, V.G. Elenskii, V.Yu. Timoshenko, P.K. Kashkarov, Modification of the properties of porous silicon on adsorption of iodine molecules // Semiconductors, 41(8), p. 953-957 (2007).
9. L.A. Osminkina, E.A. Konstantinova, K.S. Sharov, P.K. Kashkarov, V.Yu. Timoshenko, The role of boron impurity in the activation of free charge carriers in layers of porous silicon during the adsorption of acceptor molecules // Semiconductors, 39(3), p. 347-350 (2005).

10. Yu.A. Vashpanov, Electronic properties and adsorption sensitivity to ammonia of microporous silicon // Ukr. J. Phys., 44(4), p. 468-470 (1999), in Ukrainian.

11. L.S. Monastyrskii, I.B. Olenych, O.I. Aksimentyeva, B.S. Sokolovskii, M.R. Pavlyk, Gas adsorption sensors on the basis of porous silicon // Sensor Electron. and Microsystem Technol., 2(3), p. 38-43 (2011), in Ukrainian.

12. I.B. Olenych, L.S. Monastyrskii, O.I. Aksimentyeva, B.S. Sokolovskii, Humidity sensitive structures on the basis of porous silicon // Ukr. J. Phys., 56(11), p. 1198-1202 (2011).

13. A.G. Cullis, L.T. Canham, P.D.J. Calcott, The structural and luminescence properties of porous silicon // J. Appl. Phys., 82(3), p. 909-965 (1997).

14. P.V. Galiy, T.I. Lesiv, L.S. Monastyrskii, T.M. Nenchuk, I.B. Olenych, Surface investigation of nanostructured porous silicon // Thin Solid Films, 318(1-2), p. 113-116 (1998).

15. V.I. Liashenko, V.G. Litovchenko, I.I. Stepko, V.I. Strikha, L.V. Liashenko, Electronic Phenomena on the Surface Semiconductors, Ed. V.I. Liashenko. Naukova Dumka, Kiev, 1968 (in Russian).

16. M. Chiesa, G. Amato, L. Boarino, E. Garrone, F. Geobaldo, E. Giamello, Reversible insulator-tometal transition in $p$-type mesoporous silicon induced by the adsorption of ammonia // Angewandte Chemie Intern. Edition, 42(41), p. 5032-5035 (2003). 